\title{
Prevalence and potential risk factors of urticaria in the Polish population of children and adolescents
}

\author{
Marcel Mazur, Maria Czarnobilska, Ewa Czarnobilska \\ Department of Clinical and Environmental Allergology, Jagiellonian University Medical College, Krakow, Poland \\ Adv Dermatol Allergol 2020; XXXVII (5): 785-789 \\ DOI: https://doi.org/10.5114/ada.2020.100489
}

\begin{abstract}
Introduction: Urticaria is a frequent, mast cell driven disease of great social significance as it may lead to disablement, impair quality of life and affect performance at work and school.

Aim: A study on the prevalence and potential risk factors for acute and chronic urticaria in the population of children and adolescents living in Krakow, one of the major cities in Poland.

Material and methods: In this paper we present a study on the prevalence and potential risk factors of acute and chronic urticaria in the population of children aged 7-8 years old and adolescents aged 16-17 years old living in Krakow, one of the major cities in Poland. The relation of urticaria prevalence and exposure to polluted air was also investigated. In the period of 3 years between 2016 and 2018 we enrolled 17082 participants (9287 children and 7795 adolescents).

Results: Lifetime occurrence of at least one episode of urticaria was reported by $3.3 \%$ of respondents (3.6\% in the children group and $2.8 \%$ in adolescents). The most frequently mentioned causative agent was medicines in children and food in adolescents. Chronic urticaria occurred in $2.1 \%$ of children and $1.1 \%$ of adolescents. We found no relationship between the volume of traffic and the distance of residence from the high-traffic street to the frequency of urticaria.

Conclusions: A higher incidence of urticaria in the younger age group may suggest an upward trend in the incidence of this disease in the near future. The incidence of chronic urticaria in children turned out to be higher than in the European population. As our research results show, the incidence of urticaria in children and adolescents does not depend on exposure to polluted air.
\end{abstract}

Key words: urticaria, epidemiology, children, adolescents.

\section{Introduction}

Urticaria is a frequent, mast cell-driven disease, presenting with wheals, angioedema, or both. The lifetime prevalence of urticaria in the general population is approximately $20 \%$ [1]. It is a disease of great social significance as it may lead to disablement, impair quality of life and affect performance at work and school [1]. Urticaria is classified based on its duration as acute ( $\leq 6$ weeks) or chronic (> 6 weeks) [1]. With no specific eliciting factor involved it is classified as spontaneous, with a specific eliciting factor involved it is regarded as inducible. Recommended classification of chronic urticaria includes chronic spontaneous urticaria (CSU) and inducible urticaria [1].
To this day, despite numerous studies, the etiopathogenesis of this disease has not been fully explained and the treatment that would be always effective has not been introduced. While more and more is known about the prevalence of urticaria in adults, there are few data on the prevalence of urticaria in children and its dependence on possible external provocative factors such as air pollution which otherwise have proven to be important in the development of atopic diseases [2, 3].

\section{Aim}

In this paper we present a study on the prevalence and potential risk factors for acute and chronic urticaria

Address for correspondence: Prof. Ewa Czarnobilska MD, PhD, Department of Clinical and Environmental Allergology, Jagiellonian University Medical College, 10 Sniadeckich St, 31-501 Krakow, Poland, phone: +48 124248898 , fax: +48 12 423 11 22, e-mail: ewa.czarnobilska@uj.edu.pl Received: 14.10.2019, accepted: 20.11.2019. 
in the population of children and adolescents living in Krakow, one of the major cities in Poland.

\section{Material and methods}

The study was a part of a larger questionnaire-based survey aimed to assess the epidemiology of allergic diseases among children and adolescents and the impact of air pollution on their occurrence. Parents of children of 7-8 years of age and adolescents aged 16-17 living and attending schools in Krakow, Poland, were asked to participate in the study. A questionnaire originally designed for the purposes of the EUP study [4] adapted to the age group included 19 closed and semi-closed questions. Questions referred to the occurrence of urticaria and/or angioedema episodes in participants and causative factors that, in the respondents' opinion, could have contributed to their occurrence (Appendix 1). We also asked respondents about the amount of traffic on the road where they live, and if the volume of traffic was high, how far away they live (Appendix 2). In the children group, parents were answering the questions. In the adolescents group, the participants answered the questions themselves. In the period of 3 years between 2016 and 2018 we enrolled 17082 participants (9287 children and 7795 adolescents) who completed full sets of questionnaire. The analysis of the occurrence of urticaria was conducted in the whole group. The study was approved by the Ethics Committee of the Jagiellonian University.

\section{Results}

Lifetime occurrence of at least one episode of urticaria was reported by $3.3 \%$ of respondents. Interestingly the prevalence of urticaria was higher in the children group (3.6\%) than in adolescents (2.8\%).

A single episode of urticaria was reported by $2.3 \%$ of child participants while $1.7 \%$ reported repeated episodes. The average duration of an urticaria episode was 7.5 weeks. Chronic urticaria occurred in $2.1 \%$. Mean age of the first episode was 3.3 years (Tables 1 and 2). The most frequently mentioned causative agent was medicines, followed by food and contact with plants (Table 3).
The incidence of single and repeated urticarial episodes in the adolescents group was $1.3 \%$ and $1.2 \%$, respectively. The average duration of urticaria episode was 4.8 weeks. Chronic urticaria affected $1.1 \%$. Mean age of the first episode was 7.7 years (Tables 2 and 4). The most frequently mentioned causative agent was food, followed by contact with plants and medicines (Table 3).

Urticaria was more common in females than in males (1.9 vs. $1.7 \%$ in children and 1.8 vs. $1.0 \%$ in adolescents).

Traffic volume as well as the distance of the road with heavy traffic had no effect on the overall incidence of urticaria nor the incidence of chronic urticaria (the significance level was determined using the $\chi^{2}$ test). Analysis of the lifetime incidence of urticaria in respondents living close to $(<200 \mathrm{~m}$ ) roads with medium and heavy traffic showed a similar percentage in the group of 7-8-year-olds (32.3\%) and 16-17-year-olds (31.4\%) with no significant statistical difference $(p<0.89)$. No difference was found in the frequency of reports of chronic urticaria by people living close to $(<200 \mathrm{~m})$ a medium and high intensity road: $47 \%$ of $7-8$-year-olds and $48 \%$ of $16-17$-year-olds $(p<0.87)$.

\section{Discussion}

There are limited data on prevalence of urticaria in children in Poland. It is the first population-based epidemiological study to estimate the prevalence of acute and chronic urticaria in children and adolescents in Poland living in the urban, polluted area.

In Europe urticaria is rarely encountered in childhood (3.4\% the UK, 4.4\% Germany, 5.4\% Denmark) and chronic urticaria is even less probable (0.1-0.3\%) [5]. However, according to recent data, the prevalence of chronic urticaria in children in other parts of the world is higher $(0.7 \%$ chronic continuous urticaria and $1.1 \%$ chronic recurrent urticaria) [6-8].

The overall incidence of urticaria in children and adolescents observed in our study is similar to that of the European population [5]. Yet a higher incidence of urticaria in the younger age group may suggest an upward trend in the incidence of this disease in the near future.

Table 1. Urticaria symptomatology in the group of 7-8-year-olds

\begin{tabular}{|c|c|c|c|c|c|c|c|}
\hline Parameter & $\begin{array}{l}\text { Urticaria lifetime } \\
\text { prevalence }\end{array}$ & Hives & Itching & Other & $\begin{array}{c}\text { One } \\
\text { episode }\end{array}$ & $\begin{array}{c}\text { Many } \\
\text { episodes }\end{array}$ & $\begin{array}{l}\text { Duration } \\
>6 \text { weeks }\end{array}$ \\
\hline Positive number & 338 & 250 & 208 & 62 & 215 & 157 & 194 \\
\hline Percentage & 3.6 & 2.7 & 2.2 & 0.6 & 2.3 & 1.7 & 2.1 \\
\hline Number of positive among boys & 160 & 112 & 92 & 32 & 111 & 60 & 94 \\
\hline$\%$ & 1.7 & 1.2 & 1.0 & 0.3 & 1.2 & 0.7 & 1.0 \\
\hline Number of positive among girls & 177 & 138 & 115 & 30 & 103 & 97 & 100 \\
\hline$\%$ & 1.9 & 1.5 & 1.2 & 0.3 & 1.1 & 1.0 & 1.1 \\
\hline
\end{tabular}


Table 2. Age of the first episode of urticaria

\begin{tabular}{lcccc}
\hline Age of first episode [years] & Mean & SD & Min. & Max. \\
\hline $7-8$ & 3.31 & 1.84 & 0.3 & 8 \\
\hline $16-17$ & 7.68 & 4.83 & 0.5 & 17 \\
\hline
\end{tabular}

Table 3. Urticaria causative factors

\begin{tabular}{lccc}
\hline Causative factor & Medicines $(\%)$ & Food $(\%)$ & Contact with plants $(\%)$ \\
\hline $7-8$ years & 1.5 & 1.3 & 1.0 \\
\hline $16-17$ years & 0.6 & 0.9 & 0.8 \\
\hline
\end{tabular}

Table 4. Urticaria symptomatology in the group of 16-17-year-olds compared to 7-8-year-olds

\begin{tabular}{|c|c|c|c|c|c|c|c|}
\hline Parameter & $\begin{array}{c}\text { Urticaria lifetime } \\
\text { prevalence }\end{array}$ & Hives & Itching & Other & $\begin{array}{c}\text { One } \\
\text { episode }\end{array}$ & $\begin{array}{c}\text { Many } \\
\text { episodes }\end{array}$ & $\begin{array}{c}\text { Duration } \\
>6 \text { weeks }\end{array}$ \\
\hline Positive number & 218 & 86 & 116 & 17 & 104 & 91 & 82 \\
\hline$\%$ & 2.8 & 1.1 & 1.5 & 0.2 & 1.3 & 1.1 & 1.1 \\
\hline$P$-value $\left(\chi^{2}\right)$ & 0.003 & 0.0001 & 0.0004 & 0.0001 & 0.0001 & 0.005 & 0.0001 \\
\hline Number of positive among boys & 80 & 26 & 33 & 3 & 40 & 26 & 27 \\
\hline$\%$ & 1.0 & 0.3 & 0.4 & 0.03 & 0.5 & 0.3 & 0.3 \\
\hline Number of positive among girls & 138 & 60 & 83 & 14 & 64 & 65 & 55 \\
\hline$\%$ & 1.8 & 0.8 & 1.1 & 0.17 & 0.8 & 0.8 & 0.8 \\
\hline
\end{tabular}

The incidence of chronic urticaria in children turned out to be higher than in European and Asian population $[5,9]$. However, these studies concerned the general population of children, while in our study we analysed the incidence of urticaria in respondents living in a polluted urban area.

The relationship between the incidence of chronic urticaria and exposure to air pollution remains to be clarified. There is little data on the relationship of chronic urticaria with air pollutants, exposure to secondary smoke, and other life style factors that have an established importance in allergic diseases [6]. There have been findings demonstrating associations between ambient air pollution and acute urticaria [10, 11].

Air pollution, which is not observed in Western Europe to such an extent, is a serious problem of large urban areas in Poland [12]. As our research results show, living in a polluted environment (exposure to air pollution) does not seem to affect incidence of urticaria in children and adolescents.

The obtained data also indicate the threat that the use of medicines may carry. Use of drugs turned out to be the most common factor associated with the occurrence of urticaria in children. Drugs are usually not listed first as a trigger in this age group [7, 8]. One of possible explanations can be the increased frequency of drug use in the Polish population of children or the co-occurrence of infection and drug use [13].
In the group of teenagers, the most frequently reported factor that caused urticaria was food. Food may elicit urticaria or augment disease activity. These reactions are mostly of pseudoallergic nature, directed against artificial additives as well as naturally occurring aromatic components [14]. Further research should determine whether primary food allergens or an allergy to food additives cause these reactions in a young age group.

\section{Conclusions}

There is a need for future studies to be conducted, which would explore the relationship of urticaria and extrinsic factors further. We plan to perform immunological tests that may assist in understanding more clearly the pathogenesis of chronic urticaria, its relation with factors such as air pollution, food or drug ingestion as well as in exploring the ways in which urticaria prevalence could be reduced.

\section{Acknowledgments}

The paper was done with the financial support of the N41/DBS/000321 statutory grant.

\section{Conflict of interest}

The authors declare no conflict of interest. 


\section{References}

1. Zuberbier T, Aberer W, Asero R, et al. The EAACI/GA2LEN/ EDF/WAO guideline for the definition, classification, diagnosis and management of urticaria. Allergy 2018; 73: 1393-414.

2. Hassoun Y, James C, Bernstein DI. The effects of air pollution on the development of atopic disease. Clin Rev Allergy Immunol 2019 Feb 26. doi: 10.1007/s12016-019-08730-3.

3. Slama A, Śliwczyński A, Woźnica J, et al. Impact of air pollution on hospital admissions with a focus on respiratory diseases: a time-series multi-city analysis. Environ Sci Pollut Res 2019; 26: 16998-7009.

4. Raciborski F, Kłak A, Czarnecka-Operacz M, et al. Epidemiology of urticaria in Poland - nationally representative survey results. Adv Dermatol Allergol 2018; 35: 67-73.

5. Kudryavtseva AV, Neskorodova KA, Staubach P. Urticaria in children and adolescents: an updated review of the pathogenesis and management. Pediatr Allergy Immunol 2019; 30: 17-24.

6. Lee SJ, Ha EK, Jee HM, et al. Prevalence and risk factors of urticaria with a focus on chronic urticaria in children. Allergy Asthma Immunol Res 2017; 9: 212-9.

7. Godse K, Tahiliani H, Gautam M, et al. Management of urticaria in children. Indian J Paediatr Dermatol 2014; 15: 105-9.

8. Tsakok T, Du Toit G, Flohr C. Pediatric urticaria. Immunol Allergy Clin North Am 2014; 34: 117-39.

9. Balp MM, Weller K, Carboni V, et al. Prevalence and clinical characteristics of chronic spontaneous urticaria in pediatric patients. Pediatr Allergy Immunol 2018; 29: 630-6.

10. Kousha T, Valacchi G. The air quality health index and emergency department visits for urticaria in Windsor, Canada. J Toxicol Environ Health A 2015; 78: 524-33.

11. Xu F, Yan S, Wu M, et al. Ambient ozone pollution as a risk factor for skin disorders. Br J Dermatol 2011; 165: 224-5.

12. Zieliński E, Wielgus A, Dreliszak J, Zukow W. Air pollution selected health effects in Poland. J Educ Health Sport 2018; 8: 641-8.

13. Mortureux $P$, Léauté-Labrèze $C$, Legrain-Lifermann $V$, et al Acute urticaria in infancy and early childhood. Arch Dermatol 1998; 134: 319-23.

14. Zuberbier T. Role of food in urticaria. Medicine 2007 doi:10.1594/eaacinet2007/EO/6-300507. 


\section{Appendix 1. Survey questions (English translation)}

1. Have you ever had any symptoms of urticaria?

2. What symptoms of urticaria have you ever observed?

3. Have those symptoms of urticaria occurred once or repeatedly in your life?

4. How often, on average, do you experience the symptoms of urticaria?

5. Has urticaria ever lasted for longer than 6 weeks?

6. What was the duration of the longest period of urticaria symptoms?

7. At what age did you have the first episode of urticaria?

8. What factor causes your urticaria? If you don't know, please tell what you suspect.

9. Have you ever experienced angioedema?

10. Was it in the course of urticaria?

11. Have you ever had problems with breathing in the course of urticaria or angioedema?

12. Have you consulted a doctor on the symptoms of urticaria or angioedema?

13. What doctors have you consulted on the symptoms of urticaria or angioedema?

14. Did the doctor give you any medications for symptoms of urticaria or angioedema?

15. What medications for symptoms of urticaria or angioedema did you receive from the doctor?

16. Have you ever been to a hospital/hospital emergency room due to urticaria or angioedema?

17. When was the last time that you had urticaria or angioedema?

18. Are you currently on any medications due to urticaria or angioedema?

19. Please try to assess on a scale of 1 to 10 the negative influence of urticaria or angioedema on your life when symptoms appear.

\section{Appendix 2. Road traffic questions (English translation)}

1. How would you rate the amount of traffic on the road where the child lives/you live?
A. None
B. Small
C. Medium
D. Large

2. How far does the child/do you live from the road with a lot of traffic?
A. $\leq 200 \mathrm{~m}$
B. $>200 \mathrm{~m}$ 